\title{
A THREE-DIMENSIONAL SIMULATION AND VISUALIZATION SYSTEM FOR UAV PHOTOGRAMMETRY
}

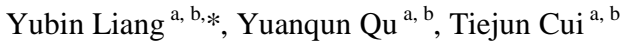 \\ ${ }^{a}$ College of Urban and Environmental Sciences, Tianjin Normal University, Tianjin 300387, China \\ ${ }^{b}$ Tianjin Engineering Center for Geospatial Information Technology, Tianjin Normal University, Tianjin 300387, China \\ lyb.whu@gmail.com; 892359961@qq.com; tiejun_cui@163.com
}

\section{ICWG I/II}

KEY WORDS: UAV Photogrammetry, Flight Planning, Image Matching, Direct Georeferencing, Global Digital Elevation Model (GDEM), Aerial Triangulation

\begin{abstract}
:
Nowadays UAVs has been widely used for large-scale surveying and mapping. Compared with manned aircraft, UAVs are more cost-effective and responsive. However, UAVs are usually more sensitive to wind condition, which greatly influences their positions and orientations. The flight height of a UAV is relative low, and the relief of the terrain may result in serious occlusions. Moreover, the observations acquired by the Position and Orientation System (POS) are usually less accurate than those acquired in manned aerial photogrammetry. All of these factors bring in uncertainties to UAV photogrammetry. To investigate these uncertainties, a three-dimensional simulation and visualization system has been developed. The system is demonstrated with flight plan evaluation, image matching, POS-supported direct georeferencing, and ortho-mosaicing. Experimental results show that the presented system is effective for flight plan evaluation. The generated image pairs are accurate and false matches can be effectively filtered. The presented system dynamically visualizes the results of direct georeferencing in three-dimensions, which is informative and effective for real-time target tracking and positioning. The dynamically generated orthomosaic can be used in emergency applications. The presented system has also been used for teaching theories and applications of UAV photogrammetry.
\end{abstract}

\section{INTRODUCTION}

Nowadays UAVs has been widely used for large-scale surveying and mapping (Haala et al., 2011; Remondino et al., 2011; Colomina and Molina, 2014). Compared with manned aircraft, UAVs are more cost-effective and more responsive. However, the POS data acquired in UAV photogrammetry are usually less accurate than those acquired in manned aerial photogrammetry. UAVs are usually more sensitive to weather condition, i.e. wind greatly influences positions and orientations of UAVs. In addition, the relief of the terrain may result in serious occlusions. All of these factors bring in uncertainties to flight planning, image matching, direct georeferencing and ortho-mosaicing, etc.

Flight planners usually consider the average elevation of the whole surveying area. However, the relative flight heights of UAVs are usually lower than those of manned aircrafts. The complexity of the terrain may result in serious occlusions and under-overlap due to low flight heights.

The SIFT operator proposed by Lowe (2004) has been widely used for matching images with large rotation, scale and lighting variance (Snavely et al., 2006; Agarwal et al., 2011). Keypoint descriptors indexed by approximate nearest neighbors algorithm (Arya et al., 1998) were used to determine tie points. Outliers from initial matches were removed using the fundamental matrix (Hartley and Zisserman, 2004) with RANSAC (Fischler and Bolles, 1981). Although the epipolar geometry guarantees strict geometric relationship between tie points, there are still situations where this framework results in false matches. The false matches may bring in errors and unnecessary computations to the following relative orientation procedure. It has been reported that researchers used GPS/POS data in image matching (Yuan and Ming, 2010). Footprints and Voronoi diagrams have been used to generate image pairs. Footprint-based method generated the footprints images and then determined the image pairs by footprint intersection (Rupnik et al., 2014). The terrain is usually assumed planar for this kind of methods. If the terrain is complex, the footprints will not lie on a plane, which makes it difficult to determine the intersections.

Another method to generate image pairs is based on the Voronoi diagram (Zhang al., 2009). However, the Voronoi diagram generated by the horizon positions of exposures only determines the neighboring relations among images. Neighboring images do not necessarily overlap, which may still leave unnecessary computations to the relative orientation.

Nowadays, global digital elevation models are freely available on the web and widely used in navigation, hydrology, and geology, etc. Rexer and Hirt (2014) evaluated the accuracy of the ASTER GDEM2 and SRTM v2.1/v4.1 over Australia, and found that the elevation accuracy of these datasets were at the level of meters. The high accuracy of the datasets enables applications such as satellite photogrammetry (Teo et al., 2010).

POS data can be used for direct georeferencing (Baumker and Heimes, 2001; Zhao et al., 2014). The 3D position of a ground point observed by more than two images can be determined by

\footnotetext{
* Corresponding author
} 
using spatial intersection. Image observations should be obtained by image matching. If an image point is not reliably matched, its spatial position cannot be determined using this method. DEM can also be used for direct georeferencing. DEMbased direct georeferencing usually starts from an average elevation and iterates to converge. However, the iterative method may stop at a local optimal with bad initialization due to complex terrain and inaccurate POS data.

A three-dimensional simulation and visualization system for UAV photogrammetry has been developed. In this paper, the system is demonstrated with flight plan evaluation, image matching, POS-supported direct georeferencing and orthomosacing. Section 2 details the methodology of core utility programs of the proposed system. Experimental results are discussed in Section 3 and conclusion is made in Section 4.

\section{METHODOLOGY}

The proposed system is designed and implemented in $\mathrm{B} / \mathrm{S}$ mode. On the server side, there is a set of utility programs implemented in $\mathrm{C}++$. The utility programs are responsible for generation of simulation data, perspective projection, coordinate transformation, file $\mathrm{I} / \mathrm{O}, \mathrm{DB}$ access and real-time communication, etc. The $3 \mathrm{D}$ visualization on the browser side is implemented in JavaScript and uses the open-source JavaScript library Cesium as its visualization engine. The presented system runs on a Dell E6440 laptop with 8GB main memory and 64-bit Windows 7 operating system

Perspective projection in this work is based on the collinearity equation, which is mainly used to generate the image observations of the ground sample points. The collinearity equation is a mathematical model which projects a three dimensional point $\mathrm{P}$ onto the image plane. Figure 1 illustrates the geometric configuration of perspective projection defined by the collinearity equation (equation (1)).

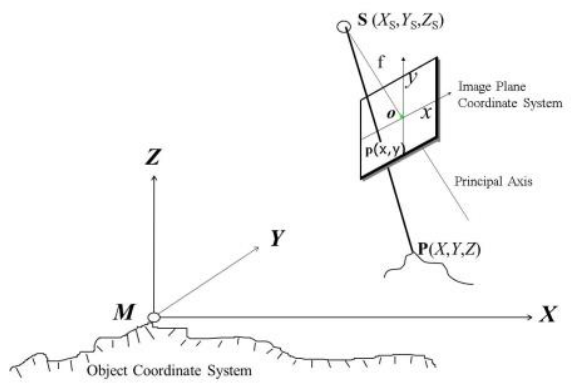

Figure 1. The geometric configuration of perspective projection defined by the collinearity equation

$$
\left.\begin{array}{l}
x=-f \frac{a_{1}\left(X-X_{S}\right)+b_{1}\left(Y-Y_{S}\right)+c_{1}\left(Z-Z_{S}\right)}{a_{3}\left(X-X_{S}\right)+b_{3}\left(Y-Y_{S}\right)+c_{3}\left(Z-Z_{S}\right)} \\
y=-f \frac{a_{2}\left(X-X_{S}\right)+b_{2}\left(Y-Y_{S}\right)+c_{2}\left(Z-Z_{S}\right)}{a_{3}\left(X-X_{S}\right)+b_{3}\left(Y-Y_{S}\right)+c_{3}\left(Z-Z_{S}\right)}
\end{array}\right\}
$$

where $(\mathrm{X}, \mathrm{Y}, \mathrm{Z})$ is the position of a three dimensional point $\mathrm{P}$ under the object coordinate system M-XYZ, (x, y) is the position of $\mathrm{p}$ under the image plane coordinate system o-xy, $\left(\mathrm{X}_{\mathrm{S}}\right.$, $\mathrm{Y}_{\mathrm{S}}, \mathrm{Z}_{\mathrm{S}}$ ) is the position of the center of projection $\mathrm{S}$ under $\mathrm{M}$ $\mathrm{XYZ}, \mathrm{f}$ is the focal length, and the nine parameters (a1-c3) are the elements of the rotation matrix $\mathrm{R}$ defined as

$$
R=\left[\begin{array}{lll}
a_{1} & a_{2} & a_{3} \\
b_{1} & b_{2} & b_{3} \\
c_{1} & c_{2} & c_{3}
\end{array}\right]
$$

where

$$
\left.\begin{array}{l}
a_{1}=\cos \varphi \cos \kappa-\sin \varphi \sin \omega \sin \kappa \\
a_{2}=-\cos \varphi \sin \kappa-\sin \varphi \sin \omega \cos \kappa \\
a_{3}=-\sin \varphi \cos \omega \\
b_{1}=\cos \omega \sin \kappa \\
b_{2}=\cos \omega \cos \kappa \\
b_{3}=-\sin \omega \\
c_{1}=\sin \varphi \cos \kappa+\cos \varphi \sin \omega \sin \kappa \\
c_{2}=-\sin \varphi \sin \kappa+\cos \varphi \sin \omega \cos \kappa \\
c_{3}=\cos \varphi \cos \omega
\end{array}\right\}
$$

where $\varphi$ is the primary rotation about the $\mathrm{Y}$-axis, $\omega$ is the secondary rotation about the $\mathrm{X}$-axis, and $\kappa$ is the tertiary rotation about the $\mathrm{Z}$-axis.

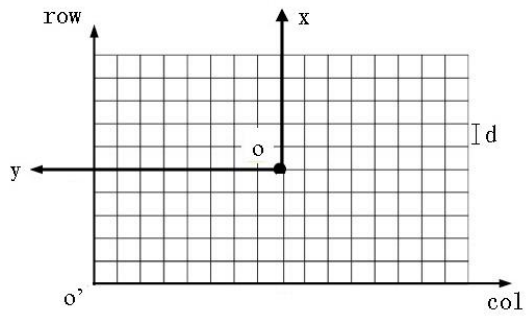

Figure 2. The image plane coordinate system and pixel coordinate system

When all of the parameters in equation (1) are known, the projection $\mathrm{p}$ of a ground point $\mathrm{P}$ can be determined. Given the relationship between the image plane coordinate system o-xy and the pixel coordinate system o'-colrow shown in Figure 2, the position of $\mathrm{p}$ under o'-colrow can be determined by equation (4).

$$
\left.\begin{array}{l}
c o l=\operatorname{col} l_{p p}-\frac{y}{d} \\
r o w=\operatorname{row}_{p p}+\frac{x}{d}
\end{array}\right\}
$$

where $\left(\mathrm{col}_{\mathrm{pp}}, \mathrm{row}_{\mathrm{pp}}\right)$ is the position of the principal point under $\mathrm{o}$-colrow, $(\mathrm{x}, \mathrm{y})$ is the position of $\mathrm{p}$ under $\mathrm{o}$-xy, and $\mathrm{d}$ is the pixel size.
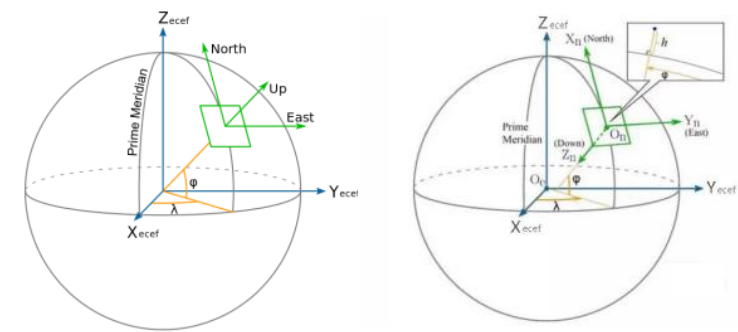

Figure 3. The ENU and NED coordinate system

Perspective projection in the proposed system is computed under the East-North-Up (ENU) coordinate system (Figure 3). The ground sample points can be simulated or sampled from the GDEM. When the range of the research area and GSD are given and the terrain is largely planar, the ground sample points can be generated randomly. If the terrain is complex, the ground sample points should be sampled from the GDEM data and the 
sample points should be transformed to ENU. In the proposed system we use the ASTER GDEM 2 in favour of its high resolution and accuracy.

The data acquired by the POS consists of position and orientation observations. The position observations are usually latitude, longitude and altitude which are defined under the geodetic coordinate system, and can be transformed directly to ENU. The orientation observations are usually pitch, roll and yaw which are defined under the North-East-Down (NED) coordinate system, and can be used to construct the rotation matrix $\mathrm{R}$ in equation (2).

The direct georeferencing in this work is based on the following equation.

$$
\left.\begin{array}{l}
X=X_{S}+\left(Z-Z_{S}\right)\left(a_{1} * x+a_{2} * y-a_{3} * f\right) /\left(c_{1} * x+c_{2} * y-c_{3} * f\right) \\
Y=Y_{S}+\left(Z-Z_{S}\right)\left(b_{1} * x+b_{2} * y-b_{3} * f\right) /\left(c_{1} * x+c_{2} * y-c_{3} * f\right)
\end{array}\right\}
$$

where all the variables in the equation above are the same as those in equation (1). The $3 \mathrm{D}$ position of $\mathrm{p}$ under $\mathrm{M}-\mathrm{XYZ}$ can be determined with the following procedure.

a. Given an image point $\mathrm{p}$, the interior/exterior orientation parameters, and the DEM that covers the research area.

b. Resample the DEM under ENU and obtain the minimum $Z_{\text {min }}$ and maximum $Z_{\max }$ of $Z$ coordinates of all the sample points.

c. Given the incremental step $\Delta Z$, iterate through all the $Z_{i}$ in the range of $\left[Z_{\min }, Z_{\max }\right]$. Determine the horizontal position $(\mathrm{X}, \mathrm{Y})$ of $p$ using equation (5) and test consistency of horizontalvertical coordinates using the resampled DEM. If the test is passed, store the 3D point $\mathrm{P}(\mathrm{X}, \mathrm{Y}, \mathrm{Z})$ as a candidate.

$\mathrm{d}$. The $3 \mathrm{D}$ point $\mathrm{P}$ in the set of candidate points that is nearest to the center of projection $\mathrm{S}$ is considered as the 3D position of $\mathrm{p}$. The procedure is illustrated in Figure 4.

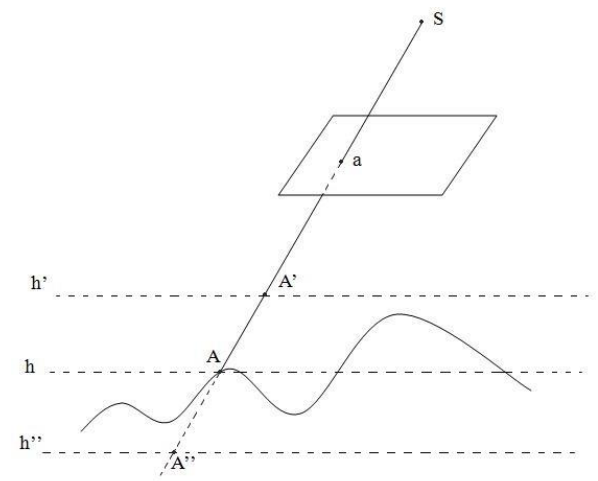

Figure 4. Illustration of the direct georeferencing

It can be seen from Figure 4 that the bundle passing the center of projection $\mathrm{S}$ and the image point a intersects with planes (h, h' and h'") with different heights at different positions (A, A' and A"). Only the true position A lie on the DEM, which means the horizontal position of $\mathrm{A}$ is consistent with its vertical position.

The results of the direct georeferencing are transformed back to the geodetic coordinate system and stored in the DB. The front end queries the DB at constant time intervals and visualizes results in the browser.
The ortho-mosaicing utility program in the current system directly uses the position and orientation data acquired by the POS. Images are firstly ortho-rectified using the DEM and then merged to the orthomosaic.

\section{RESULTS AND DISCUSSION}

The presented system is first tested with data collected in a mountainous area. The research area is visualized using the proposed system (Figure 5). The rectangle-like area is $6 \mathrm{~km}$ from east to west and $7 \mathrm{~km}$ from south to north. The elevation of the area is in the range of $[200,1050]$ meters.

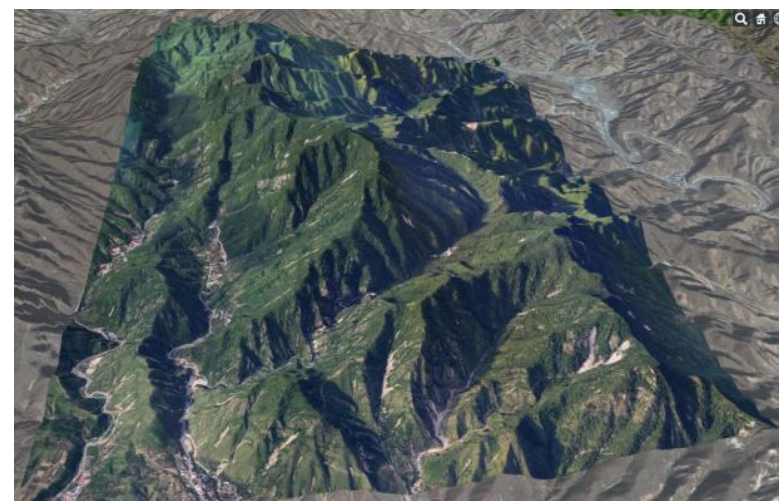

Figure 5. 3D visualization of the research area

The images were acquired with a Nikon D810 camera mounted on a fixed-wing UAV during a flight in 2016. The flight height was about 1500 meters above the sea level. After the flight, the images and POS data were downloaded. Several ground control points with accuracy at the level of decimetres were measured by using the static networked GPS.

Figure 7 and 8 shows the perspective projection of a ground control point onto an image. The true position of the ground control point is labelled with a yellow point, and the projection calculated with the POS data is labelled with a red point. It can be seen that the POS data are accurate enough to be used to locate ground control points.

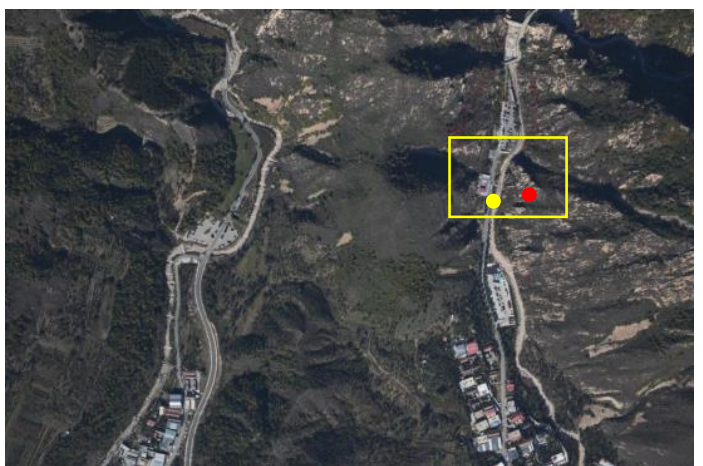

Figure 6 . The perspective projection of a ground control point on an image 


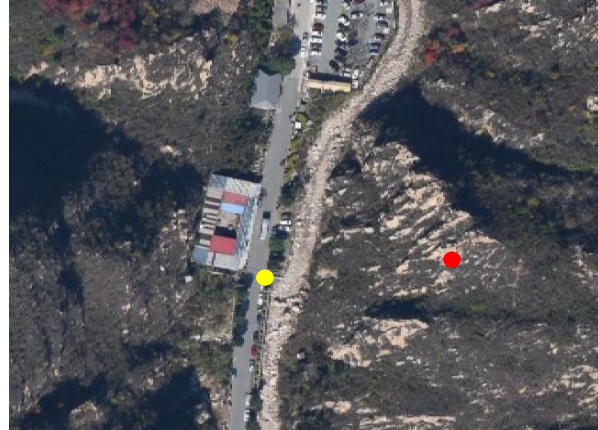

Figure 7. A closeup of the perspective projection of a ground control point

The flight plan evaluation is tested with images of the same area acquired with a Canon 5D Mark II camera during another flight in 2014. The flight height is about 1200 meters above the sea level, which means the relative flight height at the top of the mountain is less than 200 meters. The flight is designed in consideration of the average elevation of the whole area. However, the base line at the mountain top is too short to guarantee the expected forward/side overlap. The POS and DEM data are used to evaluate the flight plan. The DEM data are firstly sampled under the ENU coordinate system. Then the ground sample points are projected to the images using the POS data and imaging parameters. The images with projections of the same ground sample points are considered as overlapped. Using this method, the images that lack of overlapping are easily located. Using the exposure positions exported by the flight planner, the under-overlap problem can be discovered and avoided before the flight.

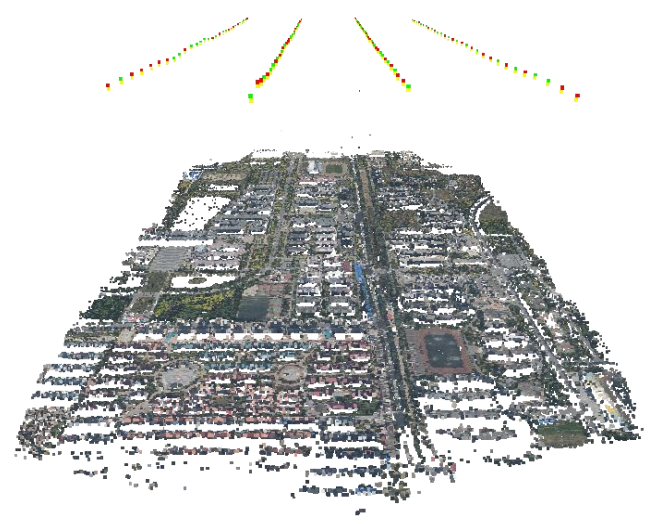

Figure 8. 120 UAV images and the reconstructed strips

Figure 8 shows the sparse reconstruction of 120 UAV images using bundler. There are 30 images along each strip. The first image is the upper right one. The initial image matching result of the first image is as follows

014283738945802758179810585761231900000

0000000180000000

000000000000000

017002070281525989159722212810370434963093

124610828746964192818400000000

000000000000000

000000000000000

$\begin{array}{lllllllllllllll}0 & 0 & 0 & 0 & 0 & 0 & 0 & 0 & 0 & 17 & 25 & 96 & 43 & 91 & 40\end{array}$
The numbers shown above is the number of tie points detected by bundler between the first image and the other images. The false matches are labelled red. The image pairs are regenerated by using the method presented in the paper. The false matches are filtered in the result.

Figure 9 shows an image acquired during the flight in 2016. The result of direct georeferencing is shown in Figure 10. The geodetic coordinates of the principal point $\mathrm{pp}$ and four corners are determined. The coordinates are exported and labelled in Google Earth to make a comparison. It can be seen from Figure 9 and 10 that area shown in the UAV image and that determined with direct georeferencing is largely overlapped. The geodetic position of the pp shown in Figure 10 corresponds well to the center of the UAV image. It should be noticed that there's a shift to the southwest in the georeferencing result. This may result from the system error in the POS data.

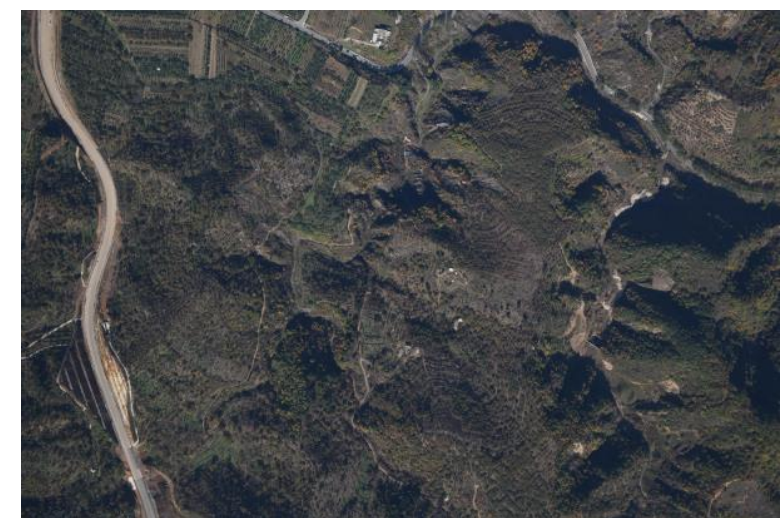

Figure 9. An UAV image from images acquired in 2016

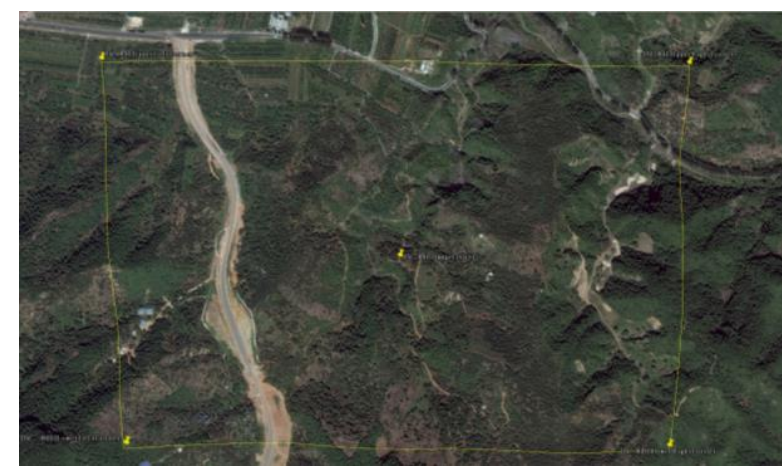

Figure 10. The direct georeferencing of an UAV image

Figure 11 shows the footprints of the images dynamically overlaid on the textured DEM in the presented system. The area covered by each image can be immediately determined. The user can navigate the area in 3D and interactively select the interest area/point and view the UAV images that cover the area/point. 


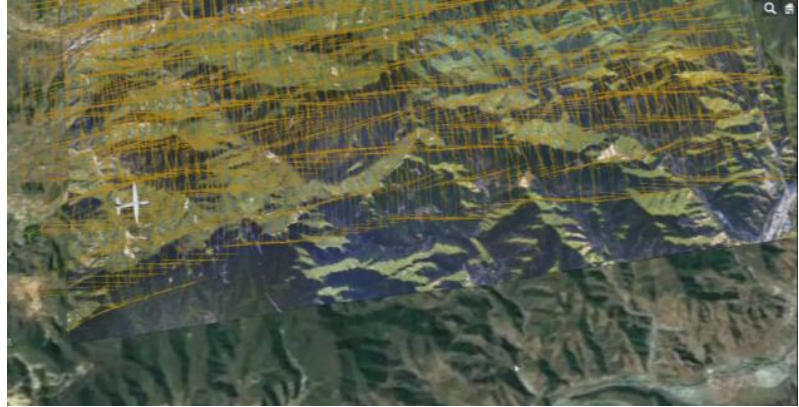

Figure 11. Dynamic visualization of the footprints

Figure 12 shows the result of real-time direct georeferencing. Two video cameras (visual and infrared) mounted on a fix-wing UAV capture high-resolution video during a flight in 2017. The video and POS data are directly transmitted to the ground station via line-of-sight communication or satellite communication. The utility programs on the server side receive the POS data and transform the coordinates and construct the rotation matrix $\mathrm{R}$ accordingly. Then the footprints of the video are generated and dynamically overlaid on the textured DEM. The blue footprints correspond to the visual camera and the red ones correspond to the infrared camera. The infrared footprints can be used to locate the burning point and measure burning area, which is very important for forest fire fighting.

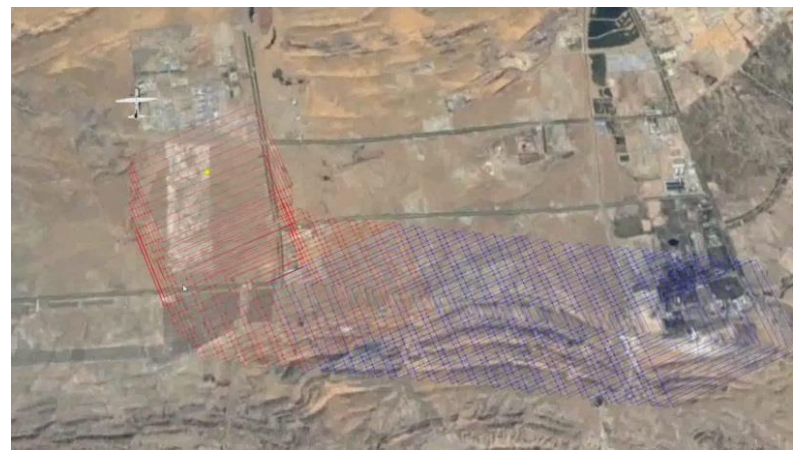

Figure 12. Real-time footprints of high-resolution video

Figure 13 shows the orthomosaic dynamically generated using the images and POS data collected during the flight in 2016. The ground sample distance of the orthomosaic is set to 1 meter. The time spent for ortho-rectification and mosaicking of each image is at the level of several seconds. An overview of the imaged area can be immediately obtained from the orthomosaic. It should be mentioned that the boundaries of neighboring images are not well merged because no bundle block adjustment has been done.

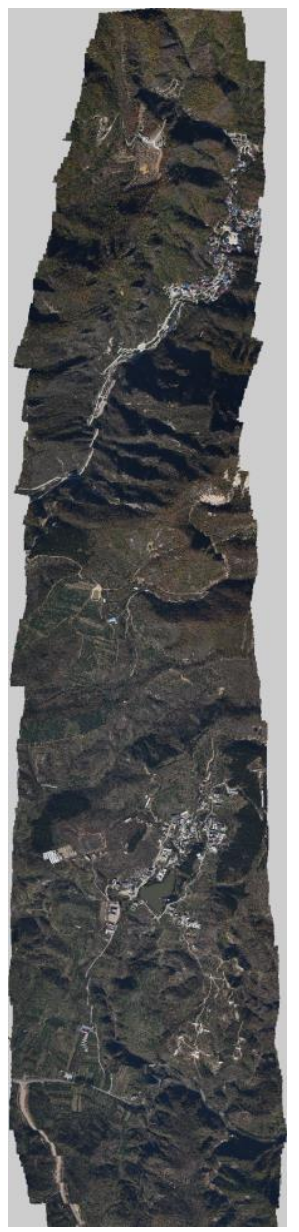

Figure 13. The dynamically generated orthomosaic

\section{CONCLUSIONS AND FUTURE WORK}

Uncertainties resulted from bad weather conditions, inaccurate POS data and complex terrain make challenges to UAV photogrammetry. A three-dimensional simulation and visualization system for UAV photogrammetry is presented. The system is demonstrated with flight plan evaluation, image matching, POS-supported direct georeferencing, and orthomosaicing. Experimental results show that well-designed algorithms could improve the robustness of UAV photogrammetry. The image pairs generated with the POS and DEM data are accurate and false matches can be effectively filtered. The image pairs are also useful for flight plan evaluation. The presented system enables 3D navigation and interaction of the results of direct georeferencing, which is useful for emergency applications.

The presented system is still under development. The future work will focus on real-time POS-supported bundle block adjustment, video ortho-mosaicing, and real-time 3D visualization of orthomosaics.

\section{ACKNOWLEDGMENT}

This work is supported by the National Natural Science Foundation of China under Grant 41471314, Municipal Key Laboratory Open Fund of Tianjin Normal University under Grant YF11700103, and the Transformation of Scientific Achievements Project of Tianjin Normal University under Grant ZX0471601116. 


\section{REFERENCES}

Agarwal, S., Furukawa, Y., Snavely, N., Simon, I., Curless, B., Seitz, S.M., Szeliski, R., 2011. Building rome in a day. Communications of the ACM 54, 105-112.

Arya, S., Mount, D.M., Netanyahu, N.S., Silverman, R., Wu, A.Y., 1998. An optimal algorithm for approximate nearest neighbor searching fixed dimensions. Journal of the ACM (JACM) 45, 891-923.

Bäumker, M., Heimes, F., 2001. New calibration and computing method for direct georeferencing of image and scanner data using the position and angular data of an hybrid inertial navigation system, OEEPE Workshop, Integrated Sensor Orientation.

Colomina, I., Molina, P., 2014. Unmanned aerial systems for photogrammetry and remote sensing: A review. ISPRS Journal of photogrammetry and remote sensing 92, 79-97.

Fischler, M.A., Bolles, R.C., 1981. Random sample consensus: a paradigm for model fitting with applications to image analysis and automated cartography. Communications of the ACM 24, 381-395.

Haala, N., Cramer, M., Weimer, F., Trittler, M., 2011. Performance test on UAV-based photogrammetric data collection. Proceedings of the International Archives of the Photogrammetry, Remote Sensing and Spatial Information Sciences 38, 7-12.

Hartley, R., Zisserman, A., 2004. Multiple view geometry in computer vision. Cambridge university press.

James, M.R., Robson, S., d'Oleire-Oltmanns, S., Niethammer, U., 2017. Optimising UAV topographic surveys processed with structure-from-motion: Ground control quality, quantity and bundle adjustment. Geomorphology 280, 51-66.

Lowe, D.G., 2004. Distinctive Image Features from ScaleInvariant Keypoints. International Journal of Computer Vision $60,91-110$.

Remondino, F., Barazzetti, L., Nex, F., Scaioni, M., Sarazzi, D., 2011. UAV photogrammetry for mapping and $3 \mathrm{~d}$ modelingcurrent status and future perspectives. International Archives of the Photogrammetry, Remote Sensing and Spatial Information Sciences 38, C22.

Rexer, M., Hirt, C., 2014. Comparison of free high resolution digital elevation data sets (ASTER GDEM2, SRTM v2. 1/v4. 1) and validation against accurate heights from the Australian National Gravity Database. Australian Journal of Earth Sciences 61, 213-226.

Rupnik, E., Nex, F., Remondino, F., 2014. Oblique multicamera systems-orientation and dense matching issues. The International Archives of Photogrammetry, Remote Sensing and Spatial Information Sciences 40, 107.

Snavely, N., Seitz, S.M., Szeliski, R., 2006. Photo tourism: exploring photo collections in 3D, ACM Transactions on Graphics (TOG). ACM, pp. 835-846.

Teo, T.A., Chen, L.C., Liu, C.L., Tung, Y.C., Wu, W.Y., 2010. DEM-Aided Block Adjustment for Satellite Images With Weak
Convergence Geometry. IEEE Transactions on Geoscience and Remote Sensing 48, 1907-1918.

Yuan, X., Ming, Y., 2010. POS-supported Matching Method for Aerial Images between Neighboring Strips. Acta Geodaetica et Cartographica Sinica 39, 156-161.

Zhang, Z., Ke, T., Guo, D., Wang, J., 2009. The appication of digital photogrammetry grid in the fast response for Wenchuan Earthquake rescue. Engineering Sciences 11, 54-62+89-93.

Zhao, H., Zhang, B., Wu, C., Zuo, Z., Chen, Z., Bi, J., 2014. Direct georeferencing of oblique and vertical imagery in different coordinate systems. ISPRS Journal of photogrammetry and remote sensing 95, 122-133.

Zhou, G., 2009. Near real-time orthorectification and mosaic of small UAV video flow for time-critical event response. IEEE Transactions on Geoscience and Remote Sensing 47, 739-747. 